\title{
Comparison of false-discovery rate for genome-wide and fine mapping regions
}

\author{
Meredith E Tabangin*1, Jessica G Woo ${ }^{1,2}$, Chunyan Liu ${ }^{1}$, Todd G Nick ${ }^{1,2}$ and \\ Lisa J Martin ${ }^{1,2}$
}

\begin{abstract}
Address: ${ }^{1}$ Department of Pediatrics, Cincinnati Children's Hospital Medical Center, 3333 Burnet Avenue, MLC 5041, Cincinnati, Ohio 452293039, USA and 'University of Cincinnati School of Medicine, 231 Albert Sabin Way, Cincinnati, Ohio 45267, USA

Email: Meredith E Tabangin* - meredith.tabangin@cchmc.org; Jessica G Woo - jessica.woo@cchmc.org; Chunyan Liu - chunyan.liu@cchmc.org; Todd G Nick - todd.nick@cchmc.org; Lisa J Martin - lisa.martin@cchmc.org

* Corresponding author
\end{abstract}

from Genetic Analysis Workshop 15

St. Pete Beach, Florida, USA. II-15 November 2006

Published: 18 December 2007

BMC Proceedings 2007, I (SuppI I):SI 48

This article is available from: http://www.biomedcentral.com/I753-656I/I/SI/SI 48

(C) 2007 Tabangin et al; licensee BioMed Central Ltd.

This is an open access article distributed under the terms of the Creative Commons Attribution License (http://creativecommons.org/licenses/by/2.0), which permits unrestricted use, distribution, and reproduction in any medium, provided the original work is properly cited.

\begin{abstract}
With technological advances in high-throughput genotyping, it is not unusual to perform hundreds of thousands of tests for each phenotype. Thus, correction to control type I error is essential. The false-discovery rate (FDR) has been successfully used in genome-wide expression data. However, its performance has not been evaluated for association analysis. Our objective was to analyze the Genetic Analysis Workshop I5 simulated data set, with answers, to evaluate FDR for genome-wide association and fine mapping. In genome-wide analysis, FDR performed well, with good localization of positive results. However, in fine mapping, all tested methods performed poorly, producing a high proportion of significant results. Thus, caution should be used when employing FDR for fine mapping.
\end{abstract}

\section{Background}

Multiple testing within research studies takes many forms, including multiple comparisons regarding a single outcome, multiple outcomes, or multiple secondary analyses. Each scenario increases the overall probability of falsepositive outcomes. With technological advances in highthroughput genotyping, it is not unusual to perform hundreds of thousands of tests for each phenotype in genomewide association studies. Thus, strategies are required to control type I error rate.
The false-discovery rate (FDR) was originally proposed by Benjamini and Hochberg as a method to adjust for multiple comparisons [1]. Less conservative than traditional control of family-wise error (FWE) rate (likelihood of making at least one type I error over all tests), FDR is the expected proportion of 'significant' tests that are truly null. FDR was extended by Storey with the $q$-value, the FDR analogue of the $p$-value [2]. The $q$-value is the proportion of significant single-nucleotide polymorphisms (SNPs) that will be false positives for a given threshold. For example, if we want to review SNPs with $q$-values $\leq$ 0.05 and this yields 200 SNPs, then approximately 10 of 
these 'significant' SNPs are expected to be null. Storey assumes that null $p$-values are uniformly distributed, and can thus be used to determine the $q$-value. These assumptions are valid for genome-wide expression data [2]. However, performance of FDR in genome-wide association studies has not been evaluated with simulated data.

In genome-wide linkage and association studies, it is common practice to follow up previously identified linkage and association signals with dense genotyping in that area (e.g., fine mapping). Genotyping a large number of SNPs in a region may introduce non-independence due to linkage disequilibrium (LD). Additionally, we would expect an increase in the number of true positives, as these regions were selected because of previous evidence of linkage or association. Unfortunately, the impact of non-independence and increased true positives on the distribution of null $p$-values and FDR performance has not been explored. Therefore, our objective was to analyze the Genetic Analysis Workshop 15 (GAW15) simulated data set, with answers, to evaluate FDR for genome-wide association and fine mapping.

\section{Methods \\ Data}

GAW15 simulated rheumatoid arthritis (RA) data consists of 1500 families with an affected sibling pair and 2000 unaffected controls. The authors had knowledge of the simulated answers at the time of analyses.

All 9187 SNPs on 22 autosomes were analyzed in 11 replicates $(90-100)$. The dense map of 17,820 SNPs on chromosome 6 was used to further model an analysis of follow-up of a known linkage peak region. Of these SNPs, 2094 within $10 \mathrm{cM}$ flanking the causative allele at locus $\mathrm{DR}$, as provided in the answers, were selected for analysis.

\section{Statistical analysis}

CCREL in the computer program R [3] was used for SNP association analysis. This package permits case-control analysis controlling for familial relationships. Because the GAW15 data provided information on family trios, as well as controls, this was an optimal method for analysis. $p$-values for each replicate were imported into QVALUE and analyzed using default settings and a FDR of 0.05 [2]. To compare FDR performance in genome-wide versus fine mapping, we compared the number of tests meeting this FDR threshold for each replicate and the average estimated $\pi_{0}$ (the probability that a given hypothesis is truly null). To compare methods, FDR was also estimated using the method of Benjamini and Hochberg [1] and a robust method assuming discrete $p$-values [4]. Results from FDR were compared with a nominal $p$-value threshold of 0.05 and Bonferroni corrected thresholds of $0.05 / 9187=5^{*} 10^{-}$
${ }^{6}$ for genome-wide and $0.05 / 2094=2 * 10^{-5}$ for fine mapping.

To examine the underlying assumption of a uniform distribution of $p$-values, we inspected the $p$-value distribution from all replicates combined. To explore the empirical false-positive (FP) rate, SNPs identified as significant in the genome-wide association analysis were examined for proximity to any of the modeled causative loci. Significant SNPs within either $10 \mathrm{cM}$ or $5 \mathrm{cM}$ of causative loci were labeled 'true positives' (TP), and the proportion of all significant SNPs labeled 'true positive' and 'false positive' (where FP = 1 - TP) was calculated.

Because non-independence could also influence the results, we examined LD between the SNP at the DR locus and 186 SNPs flanking DR. LD was calculated using $r^{2}$. Spearman's rank correlation between LD and $p$-values for the association was used to determine if the $p$-values were related to $\mathrm{LD}$.

\section{Results}

\section{Genome-wide association}

An average of $5.5 \%$ of SNPs was significant at $p \leq 0.05$ compared to $0.27 \%$ and $0.21 \%$ of SNPs using FDR and Bonferroni adjustment, respectively. Estimated $\pi_{0}$ averaged 0.99 (Table 1). The plot of $q$-values against the corresponding $p$-values exhibited exponential increases in $q$ values associated with small increases in $p$-values close to 0 (Fig. 1A). The $p$-values appeared uniformly distributed above $p=0.10$, while the frequency at $p \leq 0.05$ was only slightly higher than baseline (Fig. 2A).

FP rates for significant SNPs differed greatly by method. For the nominal $\mathrm{p} \leq 0.05$ threshold, 4920 of 5541 significant SNPs (89\%) were $>10 \mathrm{cM}$ and 5003 (90\%) were $>5$ $\mathrm{cM}$ from a causative locus. Even on chromosomes with a causative locus, the 5-cM FP rate was marginally lower on only three of the six chromosomes $(6[52 \%], 11[75 \%]$, and 18 [77\%]). Using FDR thresholds, only 9 of the 272 SNPs were $>5 \mathrm{cM}$ from a causative locus, for a 5 -cM FP rate of $3.3 \%$, with the vast majority $(96.3 \%)$ within $3 \mathrm{cM}$ of a causative locus. Bonferroni thresholds identified 217 significant SNPs, all but one of which $(0.46 \%)$ was within 3 $\mathrm{cM}$ of causative loci. Loci that mediated RA hazard or increased RA severity were not identified using any method.

\section{Fine mapping}

In contrast, using the dense chromosome 6 SNPs, an average of $45.2 \%$ of SNPs was significant at $p \leq 0.05$ compared with $46.4 \%$ and $21.4 \%$ of SNPs using FDR and Bonferroni, respectively. A cluster of 186 significant SNPs spanned $2 \mathrm{Mb}$ around $\mathrm{DR}$, but these accounted for less than $20 \%$ of significant associations. 
A
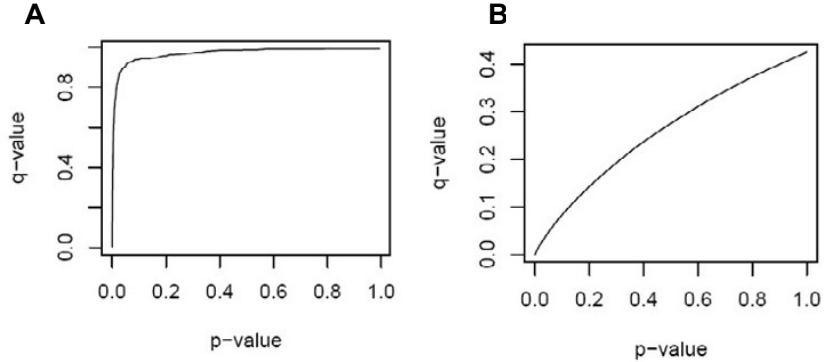

Figure I

$p$-Values versus $q$-values for all replicates combined. A, Genome-wide; B, fine mapped region.

The estimate of $\pi_{0}$ was much lower, averaging 0.41 (Table $1)$. The plot of $q$-values against the corresponding $p$-values showed nearly complete correspondence between these measures (Fig. 1B). In addition, while the $p$-values appear uniformly distributed above $p=0.10$, the frequency at $p \leq$ 0.05 is approximately 10 -fold higher than baseline (Fig. 2B). Utilization of the Benjamini and Hochberg method did not appreciably change the results (data not shown). However, using a robust FDR method reduced the number of significant results to 581 SNPs (27.7\%).

In the SNPs flanking DR, average LD $\left(r^{2}\right)$ was 0.20 , with very little correlation between $\mathrm{LD}$ and the $p$-value of the association (Spearman's $r=-0.07, \mathrm{NS}$ ).

\section{Conclusion}

Genome-wide FDR reduced the number of results identified as significant compared with unadjusted $p$-value thresholds (e.g., $p \leq 0.05$ ), and similar to Bonferroni adjusted $p$-value thresholds. Defining false positives as greater than $5 \mathrm{cM}$ from a causal locus, SNPs identified by FDR exhibited only a 3.3\% FP rate; thus, FDR was empirically more conservative than expected in this analysis.

For fine-mapped regions, FDR and p-value methods all produced a high proportion of significant results. This breakdown in FDR in fine mapping may be due to the extremely skewed distribution of $p$-values seen in the region harboring disease-causing alleles. Pounds and Cheng [4] have noted the importance of the distribution of the $p$-values in FDR estimation. When FDR was estimated using a robust methodology, there was a marked reduction in the number of significant tests; however, a large number of significant results remained. Likewise, the low correlation between pair-wise LD and p-values suggests that the large number of significant findings was not due to LD. However, because the causative locus was modeled as a tri-allelic marker, the pairwise LD between bi-allelic SNPs may not capture the true extent of LD with the DR locus.
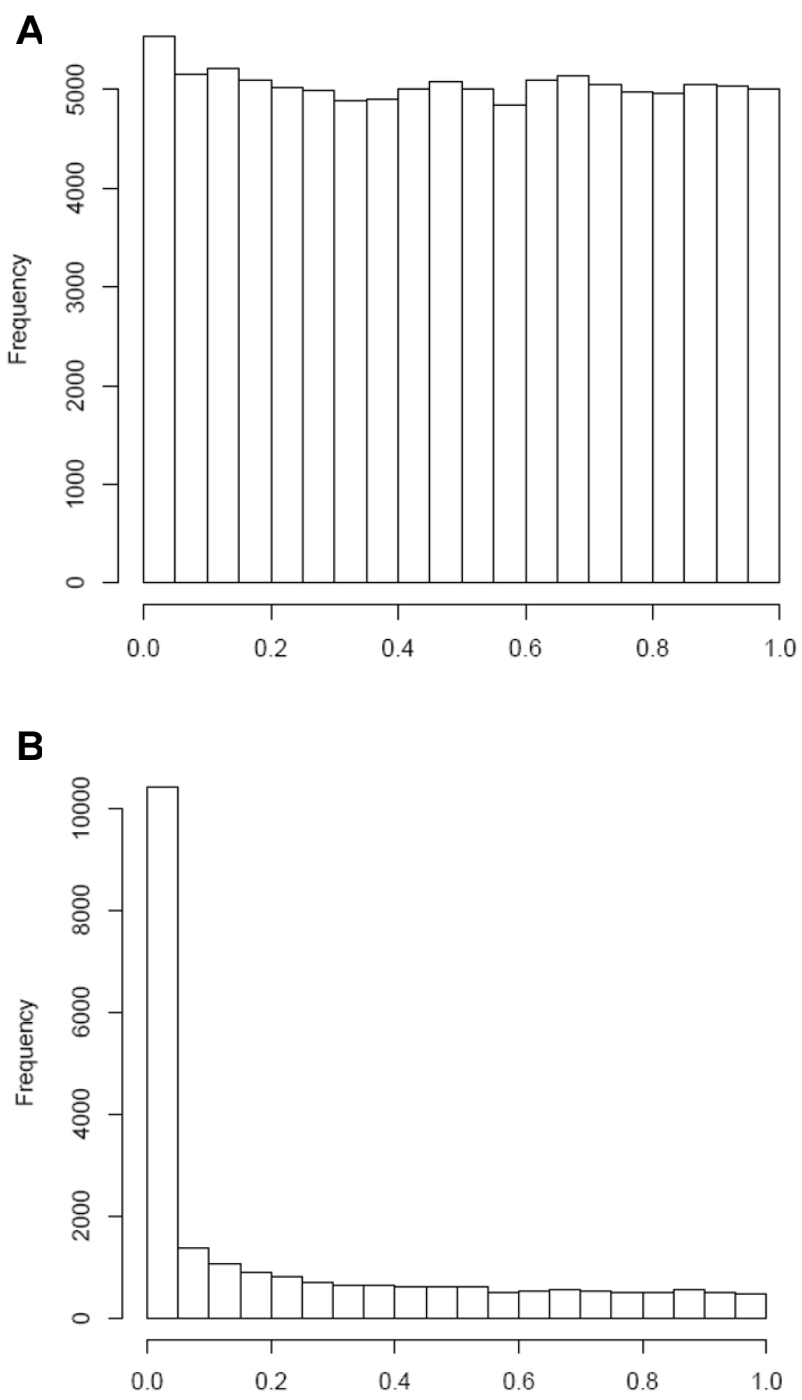

Figure 2

Histogram of $p$-values for all replicates combined. $A$, Genome-wide ( $n=9187$ SNPs per replicate); $B$, fine mapped region ( $n=2094$ SNPs per replicate).

We suspect that these findings were due to the very strong association $\left(p<10^{-300}\right)$ between the DR locus (a tri-allelic marker) and RA. Because GAW15 Problem 3 was modeled on results from RA analyses, it is possible that other diseases may exhibit similarly strong associations that persist over a large number of SNPs. This may be especially true when causal variants are multi-allelic, such as the relationship between Kringle repeat number in the apolipoprotein A gene and serum Lp(a) [5].

In summary, we have demonstrated that FDR, as implemented in the program QVALUE, appears appropriate for 
Table I: Results from FDR program QVALUE in I I replicates

\begin{tabular}{|c|c|c|c|c|c|c|c|c|}
\hline \multirow[b]{3}{*}{ Replicate } & \multicolumn{4}{|c|}{ Genome-wide ( $n=9187$ SNPs) } & \multicolumn{4}{|c|}{ Fine mapping ( $n=2094$ SNPs) } \\
\hline & \multicolumn{3}{|c|}{ Number significant (\%) } & \multirow[b]{2}{*}{ FDR $\pi_{0}$} & \multicolumn{3}{|c|}{ Number significant (\%) } & \multirow[b]{2}{*}{$\operatorname{FDR} \pi_{0}$} \\
\hline & $p \leq 0.05$ & Bonferroni & FDR & & $p \leq 0.05$ & Bonferroni & FDR & \\
\hline 90 & $498(5.4)$ & $21(0.23)$ & $28(0.30)$ & 0.982 & $944(45.1)$ & $489(23.4)$ & $100 \mid(47.8)$ & 0.339 \\
\hline 91 & $483(5.3)$ & $21(0.23)$ & $28(0.30)$ & 0.997 & $948(45.3)$ & $448(21.4)$ & $940(44.9)$ & 0.464 \\
\hline 92 & $484(5.3)$ & $23(0.25)$ & $24(0.26)$ & I & 939 (44.9) & 459 (21.9) & 1082 (5।.7) & 0.248 \\
\hline 93 & $501(5.5)$ & $18(0.20)$ & $25(0.27)$ & 1 & 914 (43.7) & $435(20.8)$ & $910(43.5)$ & 0.468 \\
\hline 94 & $526(5.7)$ & $19(0.21)$ & $23(0.25)$ & 0.989 & $899(43.0)$ & $444(21.2)$ & $879(42.0)$ & 0.471 \\
\hline 95 & $493(5.4)$ & $20(0.22)$ & $26(0.28)$ & 0.992 & $1010(48.3)$ & $432(20.6)$ & 1069 (5I.I) & 0.355 \\
\hline 96 & $494(5.4)$ & $18(0.20)$ & $26(0.28)$ & I & $945(45.2)$ & $463(22.1)$ & $966(46.1)$ & 0.411 \\
\hline 97 & $533(5.8)$ & $17(0.19)$ & $22(0.24)$ & 0.992 & $1000(47.8)$ & $443(2 \mid .2)$ & $1010(48.2)$ & 0.439 \\
\hline 98 & $500(5.4)$ & $19(0.21)$ & $22(0.24)$ & 0.958 & 939 (44.9) & 437 (20.9) & $926(44.2)$ & 0.467 \\
\hline 99 & $50 \mathrm{I}(5.5)$ & $21(0.23)$ & $26(0.28)$ & 1 & $923(44.1)$ & 459 (21.9) & 915 (43.7) & 0.486 \\
\hline 100 & $528(5.8)$ & $20(0.22)$ & $22(0.24)$ & 0.988 & $955(45.6)$ & $428(20.4)$ & 981 (46.8) & 0.366 \\
\hline Average & $503.7(5.5)$ & $19.7(0.21)$ & $24.7(0.27)$ & 0.991 & $946.9(45.2)$ & $448.8(21.4)$ & $970.8(46.4)$ & 0.410 \\
\hline
\end{tabular}

genome-wide association studies where the majority of tests will not be significant. However, caution should be employed when using it for fine mapping because these regions may contain a large number of highly significant associations. This biased distribution appears to poorly affect FDR performance. In circumstances in which a large number of significant results are identified, other approaches should be considered to control error rate such as the double trend test [6] or the use of haplotypes [7].

\section{List of abbreviations used}

FDR: False Discovery Rate

FP: False positive

LD: Linkage Disequilibrium

RA: Rheumatoid Arthritis

SNP: Single Nucleotide Polymorphism

TP: True positive

\section{Competing interests}

The author(s) declare that they have no competing interests.

\section{Acknowledgements}

This work was supported in part by NIH grants HL074I68, HL07432I, and HL74728. We thank Prakash Velayutham and Dr. Michael Wagner for their assistance in the implementation of CCREL in a parallel process.

This article has been published as part of BMC Proceedings Volume I Supplement I, 2007: Genetic Analysis Workshop I5: Gene Expression Analysis and Approaches to Detecting Multiple Functional Loci. The full contents of the supplement are available online at http://www.biomedcentral.com/ $|753-656| / \mid$ ? issue=SI.

\section{References}

I. Benjamini $Y$, Hochberg $Y$ : Controlling the false discovery rate: a practical and powerful approach to multiple testing. J R Stat Soc Ser B 1995, 57:289-300.

2. Storey JD, Tibshirani R: Statistical significance for genome-wide experiments. Proc Natl Acad Sci USA 2003, 100:9440-9445.

3. Browning SR, Briley JD, Briley LP, Chandra G, Charnecki JH, Ehm MG, Johansson KA, Jones BJ, Karter AJ, Yarnall DP, Wagner MJ: Casecontrol single marker and haplotypic association analysis of pedigree data. Genet Epidemiol 2005, 28: I I0-122.

4. Pounds S, Cheng C: Robust estimation of the false discovery rate. Bioinformatics 2006, 22:1979-1987.

5. Gavish D, Azrolan N, Breslow JL: Plasma Lp(a) concentration is inversely correlated with the ratio of Kringle IV/Kringle V encoding domains in the Apo(a) gene. J Clin Invest 1989, 84:202I-2027.

6. Song K, Lu Q, Lin X, Waterworth D, Elston RC: Genome-wide association studies using an adaptive two-stage analysis for a case-control design. BMC Proc 2007, I(Suppl I):SI 47.

7. Tang R, Wang F, Sha Q, Zhang S, Chen H-S: Genome-wide association tests by using block information in family data. $B M C$ Proc 2007, I (SuppI I):SI49.

Publish with Biomed Central and every scientist can read your work free of charge

"BioMed Central will be the most significant development for disseminating the results of biomedical research in our lifetime. "

Sir Paul Nurse, Cancer Research UK

Your research papers will be:

- available free of charge to the entire biomedical community

- peer reviewed and published immediately upon acceptance

- cited in PubMed and archived on PubMed Central

- yours - you keep the copyright

Submit your manuscript here:

http://www.biomedcentral.com/info/publishing_adv.asp
BioMedcentral 